\title{
Development of a code system DEURACS for theoretical analysis and prediction of deuteron-induced reactions
}

\author{
Shinsuke Nakayama ${ }^{1, a}$, Hiroshi Kouno ${ }^{2}$, Yukinobu Watanabe ${ }^{2}$, Osamu Iwamoto $^{1}$, Tao Ye $^{2,3}$, and Kazuyuki Ogata ${ }^{4}$ \\ 1 Nuclear Data Center, Japan Atomic Energy Agency, Ibaraki 319-1195, Japan \\ 2 Department of Advanced Energy Engineering Science, Kyushu University, Fukuoka 816-8580, Japan \\ ${ }^{3}$ Institute of Applied Physics and Computational Mathematics, Beijing 100094, China \\ ${ }^{4}$ Research Center for Nuclear Physics, Osaka University, Osaka 567-0047, Japan
}

\begin{abstract}
We have developed an integrated code system dedicated for theoretical analysis and prediction of deuteron-induced reactions, which is called DEUteron-induced Reaction Analysis Code System (DEURACS). DEURACS consists of several calculation codes based on theoretical models to describe respective reaction mechanisms and it was successfully applied to $(d, x p)$ and $(d, x n)$ reactions. In the present work, the analysis of $(d, x n)$ reactions is extended to higher incident energy up to nearly $100 \mathrm{MeV}$ and also DEURACS is applied to $(d, x d)$ reactions at 80 and $100 \mathrm{MeV}$. The DEURACS calculations reproduce the experimental doubledifferential cross sections for the $(d, x n)$ and $(d, x d)$ reactions well.
\end{abstract}

\section{Introduction}

Recently, intensive neutron sources using deuteron accelerators have been proposed for various applications such as the International Fusion Materials Irradiation Facility (IFMIF) [1] and Neutron For Science (NFS) in SPIRAL2 [2], and also medical applications such as boron neutron capture therapy (BNCT) [3] and production of radioisotopes for medical use [4,5]. In these facilities, the $(d, x n)$ reactions on light elements $(\mathrm{Li}$, $\mathrm{Be}, \mathrm{C}$, etc.) are considered as promising reactions to generate intensive neutron beams. Deuteron accelerator components consist of not only neutron converter materials but also various structure materials including $\mathrm{Al}, \mathrm{Fe}, \mathrm{Ni}$, etc. Thus, accurate and comprehensive deuteron nuclear data libraries over wide ranges of target mass number and incident energy are indispensable for the design of deuteron accelerator neutron sources. Since experimental data of deuteron-induced reactions are not necessarily enough to meet the requirement, theoretical model calculation plays an important role in deuteron nuclear data evaluation.

Under these circumstances, we have so far developed an integrated code system dedicated for theoretical analysis and prediction of deuteron-induced reactions, which is called DEUteron-induced Reaction Analysis Code System (DEURACS) [6-11]. DEURACS consists of several calculation codes based on theoretical models to describe respective reaction mechanisms characteristic of deuteron-induced reactions. Elastic breakup and nonelastic breakup reactions are calculated using the codes based on the continuum-discretized coupledchannels (CDCC) theory [12] and the Glauber model

\footnotetext{
a e-mail: nakayama.shinsuke@jaea.go.jp
}

$[13,14]$, respectively. In addition, DWUCK4 [15], which is the computer code based on a conventional zerorange distorted wave Born approximation (DWBA) is used to calculate one-nucleon transfer reactions to bound states in residual nuclei. It should be noted that the nonelastic breakup and transfer reactions were called stripping reactions to the continuum and to the bound states, respectively, in Refs. [6-11,13,14]. Finally, the contribution from pre-equilibrium and compound nucleus processes are calculated using the exciton and Hauser-Feshbach models implemented in the CCONE code $[16,17]$.

Double-differential cross sections (DDXs) of $(d, x n)$ reactions are critically important in the engineering design of neutron sources. Therefore, we have so far focused on nucleon emission from deuteron-induced reactions and examined the applicability of DEURACS to such reactions. In our previous work, DEURACS was successfully applied to analyses of DDXs for $(d, x p)$ reactions at incidenet energies of 56 and $100 \mathrm{MeV}$ [6-8], activation cross sections by $(d, p)$ reactions from threshold to $50 \mathrm{MeV}$ [7-9], and double-differential thick target neutron yields (TTNYs) from $(d, x n)$ reactions up to $50 \mathrm{MeV}[10,11]$. Note that other microscopic calculations of inclusive $(d, x p)$ reactions have been recently reported by other research groups $[18,19]$.

Recently, a new measurement of DDXs of $(d, x n)$ reactions has been performed at higher incident energy of $102 \mathrm{MeV}$ [20]. In the present work, the applicability of DEURCAS to $(d, x n)$ reactions is further investigated by analyzing the measured DDXs on ${ }^{9} \mathrm{Be}$ and ${ }^{12} \mathrm{C}$ at $102 \mathrm{MeV}$. Analyses of other reaction channels except nucleon emission is also necessary to extend the applicability. Therefore, DEURACS is used to analysis of the $(d, x d)$ reactions on ${ }^{58} \mathrm{Ni}$ at $100 \mathrm{MeV}$ and ${ }^{27} \mathrm{Al}$ at $80 \mathrm{MeV}$, and improvement of the CCONE code is discussed. 


\section{Analysis of $(d, x n)$ reactions}

\subsection{Models and methods}

In DEURACS, DDXs of inclusive $(d, x n)$ reactions are expressed by incoherent summation of the following components:

$$
\frac{d^{2} \sigma^{(d, x n)}}{d E d \Omega}=\frac{d^{2} \sigma_{E B U}}{d E d \Omega}+\frac{d^{2} \sigma_{N B U}}{d E d \Omega}+\frac{d^{2} \sigma_{p-T R}}{d E d \Omega}+\frac{d^{2} \sigma_{P E+C N}}{d E d \Omega}
$$

where $d^{2} \sigma_{E B U} / d E d \Omega, d^{2} \sigma_{N B U} / d E d \Omega, d^{2} \sigma_{p-T R} / d E d \Omega$, and $d^{2} \sigma_{P E+C N} / d E d \Omega$ correspond to DDXs for the components of elastic breakup (EBU), nonelastic breakup (NBU), proton transfer ( $p$-TR), and pre-equilibrium (PE) and compound nucleus $(\mathrm{CN})$, respectively. The EBU component is directly calculated with the CDCC method. The NBU component is calculated by the Glauber model with the same noneikonal approach as in Ref. [14]. Next, the $p$-TR component to each discrete level in residual nucleus is calculated using the zero-range DWBA code DWUCK4 [15]. The DDX of $p$-TR is obtained by smearing the calculated DWBA differential cross section at a certain angle using experimental energy resolution. It should be noted that the DDXs calculated by the Glauber model and the DWBA approach overlap with each other in the energy region corresponding to the transitions to discrete levels in the residual nuclei. Therefore, we cut off the DDXs calculated by the Glauber model in this energy region to avoid double counting.

Finally, the contribution from PE and $\mathrm{CN}$ processes is calculated by combining the Glauber model and the CCONE code. In the calculation, three different compound nuclei are considered since they can be formed by absorption of either a neutron or a proton in the incident deuteron or the deuteron itself. Since the neutron or the proton absorbed in the target nucleus by the nonelastic breakup reactions has a certain energy distribution, the DDXs for the PE and $\mathrm{CN}$ components are calculated in the following way:

$$
\begin{aligned}
\frac{d^{2} \sigma_{P E+C N}}{d E d \Omega}= & \int d E_{n} R_{n}\left(E_{n}\right) \frac{d^{2} \sigma_{P E+C N}^{(n, x n)}\left(E_{n}\right)}{d E d \Omega} \\
& +\int d E_{p} R_{p}\left(E_{p}\right) \frac{d^{2} \sigma_{P E+C N}^{(p, x n)}\left(E_{p}\right)}{d E d \Omega} \\
& +R_{d} \frac{d^{2} \sigma_{P E+C N}^{(d, x n)}}{d E d \Omega}
\end{aligned}
$$

where $E_{n}$ and $E_{p}$ are energies of the neutron and proton absorbed in the target, $R_{n}, R_{p}$, and $R_{d}$ denote the formation fractions of three different compound nuclei, which are calculated with the Glauber model, and $d^{2} \sigma_{P E+C N}^{(n, x n)} / d E d \Omega, d^{2} \sigma_{P E+C N}^{(p, x n)} / d E d \Omega$, and $d^{2} \sigma_{P E+C N}^{(d, x n)} / d E d \Omega$ are the DDXs for the PE and CN components of $(n, x n),(p, x n)$, and $(d, x n)$ reactions calculated with the CCONE code, respectively.

Each calculation model integrated in DEURACS contains some input parameters. In the CDCC method and the Glauber model, nucleon optical potentials (OPs) at half the incident deuteron energy are necessary. We have

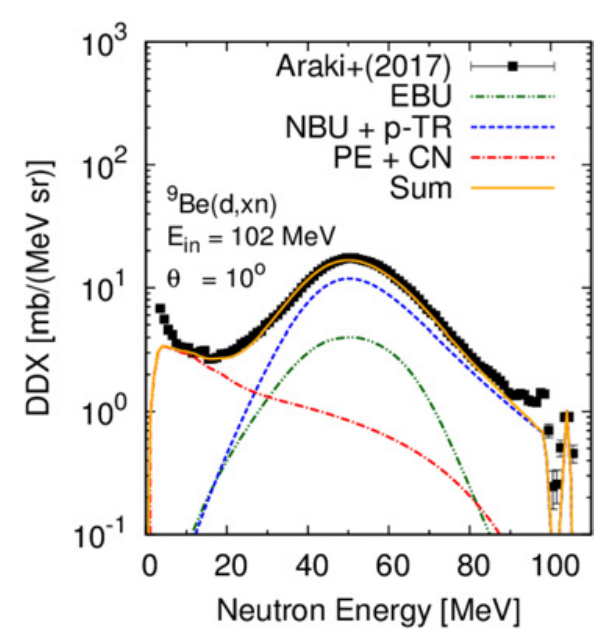

Figure 1. Calculated and experimental DDXs for the ${ }^{9} \mathrm{Be}(d, x n)$ reaction at $102 \mathrm{MeV}$. The dash-dot-dotted, the short-dashed, and the dash-dotted curves represent the components of EBU, NBU $+p$-TR, and $\mathrm{PE}+\mathrm{CN}$, respectively. The solid curves are the sum of each component. Experimental data are taken from Ref. [20].

chosen the Koning and Delaroche (K-D) OPs [21] for both neutron and proton similarly to Ref. [11].

The input parameters used in the DWUCK4 calculation are the same as those used in our previous systematic DWBA analysis [8]. In addition, DWBA calculation for the ${ }^{9} \mathrm{Be}(d, n){ }^{10} \mathrm{~B}$ and ${ }^{12} \mathrm{C}(d, n){ }^{13} \mathrm{~N}$ reactions requires the spectroscopic factors (SFs) corresponding to individual discrete levels in ${ }^{10} \mathrm{~B}$ and ${ }^{13} \mathrm{~N}$. We adopt the same SFs as those extracted in the Ref. [11]. In these SFs, a weak incident energy dependence found in Ref. [8] is introduced.

Finally, in the CCONE calculation, the An and Cai global OPs [22] and the K-D OPs are used for the deuteron OPs and the nucleon OPs, respectively. For the level-density parametrization, we use the systematics by Mengoni and Nakajima [23]. Default values in the CCONE code are employed for other input parameters.

\subsection{Results and discussion}

Figures 1 and 2 show comparisons between the calculated and experimental DDXs for the ${ }^{9} \mathrm{Be}(d, x n)$ and ${ }^{12} \mathrm{C}(d, x n)$ reactions at $102 \mathrm{MeV}$, respectively. For both target nuclei, the calculation using DEURACS reproduce well both the shape and magnitude of the experimental $(d, x n)$ spectra including the sharp peaks observed in the high emission energy region. The peaks are attribute to the $p$-TR reactions. These results demonstrate the applicability of DEURACS to the $(d, x n)$ reactions on light nuclei at incident energies around $100 \mathrm{MeV}$.

From Figs. 1 and 2, it is found that characteristic broad peak seen around half the deuteron incident energy is formed mainly by the nonelastic breakup processes. This suggests that modeling of the nonelastic breakup reactions is essential for accurate prediction of neutron yields in deuteron accelerator neutron sources. On the other hand, the DEURACS calculation underestimates the experimental data in the low energy region. As pointed out in Ref. [11], this underestimation is likely to arise from the absence of sequential neutron emission from the discrete levels of the residual nuclei. Improvement of the CCONE 


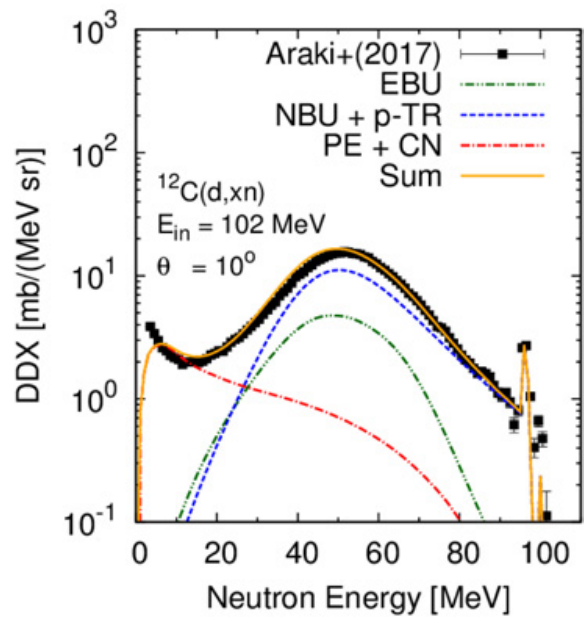

Figure 2. Same as Fig. 1 but for the ${ }^{12} \mathrm{C}(d, x n)$ reaction at $102 \mathrm{MeV}$.

code needs to be explored in our future work. In addition, the model used in the present work for the nonelastic breakup reactions may not be valid for low incident energy region close to the Coulomb barrier. Therefore, it needs to be tested in the energy region using experimental data and evaluated values such as ones reviewed and evaluated in Ref. [24].

\section{Analysis of $(d, x d)$ reactions}

In this section, we attempt to improve the CCONE code used in DEURACS so that deuteron-induced deuteron production reactions, namely $(d, x d)$ reactions, can be described reasonably well.

\subsection{Models and methods}

In DEURACS, DDXs of inclusive $(d, x d)$ reactions are expressed as follows:

$$
\frac{d^{2} \sigma^{(d, x d)}}{d E d \Omega}=\frac{d^{2} \sigma_{S-E L}}{d E d \Omega}+\frac{d^{2} \sigma_{D I R}}{d E d \Omega}+\frac{d^{2} \sigma_{P E+C N}}{d E d \Omega}
$$

where $\quad d^{2} \sigma_{S-E L} / d E d \Omega, \quad d^{2} \sigma_{D I R} / d E d \Omega, \quad$ and $d^{2} \sigma_{P E+C N} / d E d \Omega$ correspond to DDXs for the components of shape elastic scattering (S-EL), direct inelastic scattering (DIR), and pre-equilibrium (PE) and compound nucleus $(\mathrm{CN})$, respectively. The S-EL component is calculated with the optical model implemented in the CCONE code. As to the DIR component, DWBA approach with a global parametrization by Kalbach [25] is used in the CCONE code to calculate inelastic scattering cross sections for giant resonance states [26], and we adopt the approach. The direct inelastic scattering for the each discrete level in the residual nucleus is not considered.

Similarly to Eq. (2), the contribution from PE and CN processes is calculated by combining the Glauber model and the CCONE code. In the present work, we have made an improvement in CCONE. In the CCONE code, the semi-empirical inelastic scattering model proposed by Kalbach [27] is implemented. The model was originally developed for the inelastic scattering processes involving alpha particles and it expresses the effect of excitation of a nucleon in the target nucleus by the incident alpha particle. This effect is not included in the exciton model. We

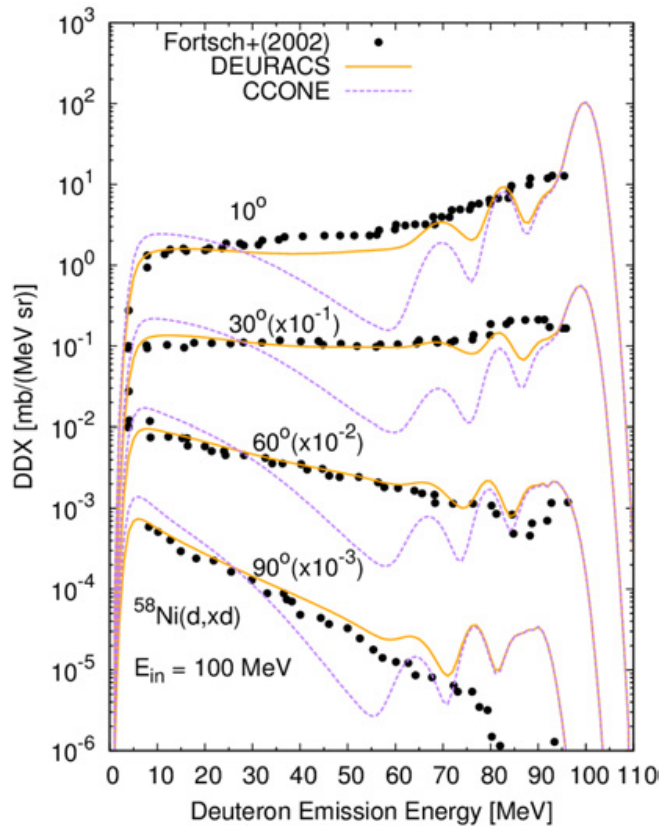

Figure 3. Calculated and experimental DDXs for the ${ }^{58} \mathrm{Ni}(d, x d)$ reaction at $100 \mathrm{MeV}$. The solid curves and the dashed lines represent results of the DEURACS calculation and those of the original CCONE code, respectively. The number at the top of each plot denotes emission angle. Experimental data are taken from Ref. [28].

extend the model to deuteron inelastic scattering processes and apply it to $(d, x d)$ reactions. In the extension, we have adopted the original model itself and the adjustable parameters used in it. Only the atomic and mass numbers of the incident and emitted particles are changed to those of deuteron inelastic scattering. The contribution from the model is added to the PE component.

Input parameters in the Glauber model and the CCONE code are the same as those used in the $(d, x n)$ reactions described in Sect. 2.1.

\subsection{Results and discussion}

Figure 3 shows comparisons between the calculated and experimental DDXs for the ${ }^{58} \mathrm{Ni}(d, x d)$ reactions at $100 \mathrm{MeV}$. In the figure, the calculation results of the original CCONE code are presented as well as those of DEURACS. The peaks seen in the highest energy region arise from the S-EL component. In addition, the broad peaks located around 65 and $80 \mathrm{MeV}$ in both calculations are attributed to the DIR component for giant resonance states. The result of analysis for the ${ }^{27} \mathrm{Al}(d, x d)$ reactions at $80 \mathrm{MeV}$ are also given in Fig. 4.

As shown in the figures, DEURACS reproduces the experimental $(d, x d)$ spectra better than the original CCONE code. Especially, cross sections in the energy region above $30 \mathrm{MeV}$ are enhanced by introducing the Kalbach's semi-empirical inelastic scattering model.

In addition, some differences between both calculations are seen in the low emission energy region. In the original CCONE code, the compound formation cross section is given by subtraction of the direct inelastic scattering cross section from the deuteron total reaction cross section. However, the incident deuteron flux is fed to other direct reaction channels such as EBU and NBU 


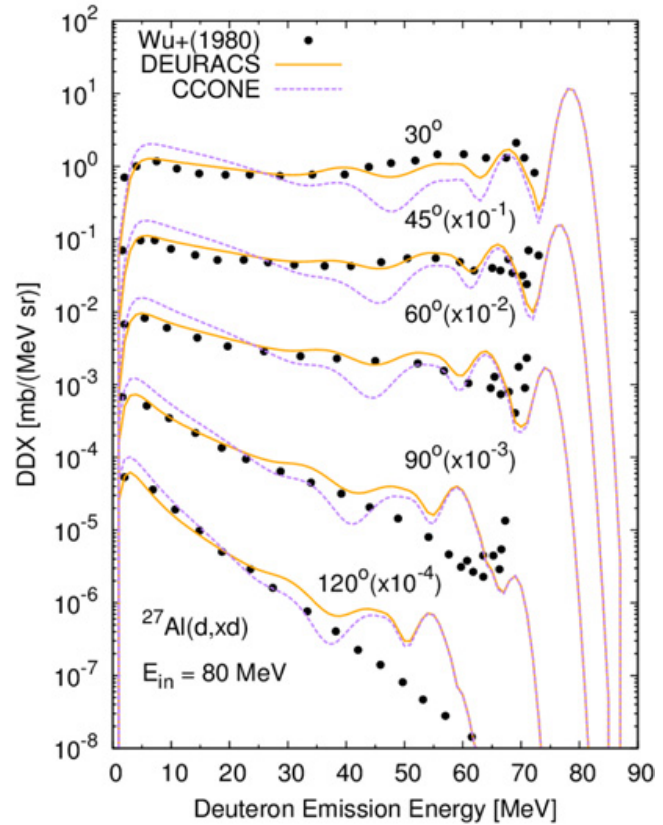

Figure 4. Same as Fig. 3 but for the ${ }^{27} \mathrm{Al}(d, x d)$ reaction at $80 \mathrm{MeV}$. Experimental data are taken from Ref. [29].

reactions. As described in Eq. (2), this effect is properly taken into account in the framework of DEURACS. This results in reduction of the cross sections of PE and $\mathrm{CN}$ processes and good agreement with the experimental DDX of $(d, x d)$ reactions at low emission energies.

\section{Summary and outlook}

We have analyzed double-differential cross sections for $(d, x n)$ and $(d, x d)$ reactions using DEUteron-induced Reaction Analysis Code System (DEURACS). In the calculation of $(d, x d)$ reactions, some improvements of the CCONE code have been made. DEURACS calculations reproduce well the experimental $(d, x n)$ and $(d, x d)$ data at the incident energies of 80 and around $100 \mathrm{MeV}$. Since we have confirmed that DEURACS is successfully applicable to deuteron-induced reactions, we plan to perform deuteron nuclear evaluation up to $200 \mathrm{MeV}$ using DEURACS in the future [30].

\section{References}

[1] A. Moeslang, V. Heinzel, H. Matsui, M. Sugimoto, Fusion Eng. Des. 81, 863 (2006)

[2] M. Fadil, B. Rannou, Nucl. Instrum. Methods Phys. Res. B 266, 4318 (2008)

[3] S. Agosteo, G. Curzio, F. d'Errico, R. Nath, R. Tinti, Nucl. Instrum. Methods Phys. Res. A 476, $106(2002)$
[4] Y. Nagai, K. Hashimoto, Y. Hatsukawa, et al., J. Phys. Soc. Jpn. 82, 064201 (2013)

[5] T. Kin, Y. Nagai, N. Iwamoto, et al., J. Phys. Soc. Jpn. 82, 034201 (2013)

[6] S. Nakayama, S. Araki, Y. Watanabe, O. Iwamoto, T. Ye, K. Ogata, Nucl. Data Sheets 118, 305 (2014)

[7] S. Nakayama, S. Araki, Y. Watanabe, O. Iwamoto, T. Ye, K. Ogata, Energy Procedia 71, 219 (2015)

[8] S. Nakayama, Y. Watanabe, J. Nucl. Sci. Technol. 53, 89 (2016)

[9] S. Nakayama, Y. Watanabe, JAEA-Conf-2015 003, 105 (2016)

[10] S. Nakayama, H. Kouno, Y. Watanabe, O. Iwamoto, T. Ye, K. Ogata, EPJ Web Conf. 122, 04004 (2016)

[11] S. Nakayama, H. Kouno, Y. Watanabe, O. Iwamoto, K. Ogata, Phys. Rev. C 94, 014618 (2016)

[12] M. Yahiro, K. Ogata, T. Matsumoto, K. Minomo, Prog. Theor. Exp. Phys. 2012, 1 A206 (2012), and references therein

[13] T. Ye, Y. Watanabe, K. Ogata, Phys. Rev. C 80, 014604 (2009)

[14] T. Ye, S. Hashimoto, Y. Watanabe, K. Ogata, M. Yahiro, Phys. Rev. C 84, 054606 (2011)

[15] P.D. Kunz, computer code DWUCK4 (unpublished), http://spot. colorado.edu/k̃unz/DWBA.html

[16] O. Iwamoto, J. Nucl. Sci. Technol. 44, 687 (2007)

[17] O. Iwamoto, N. Iwamoto, S. Kunieda, F. Minato, K. Shibata, Nucl. Data Sheets 131, 259 (2016)

[18] J. Lei, A.M. Moro, Phys. Rev. C 92, 044616 (2015)

[19] B.V. Carlson, R. Capote, M. Sin, Few-Body Syst. 57, 307 (2016)

[20] S. Araki, Y. Watanabe, M. Kitajima, et al., Nucl. Instrum. Methods Phys. Res. A 842, 62 (2017)

[21] A.J. Koning, J.P. Delaroche, Nucl. Phys. A 713, 231 (2003)

[22] H. An, C. Cai, Phys. Rev. C 73, 054605 (2006)

[23] A. Mengoni, Y. Nakajima, J. Nucl. Sci. Technol. 31, 151 (1994)

[24] S.M. Qaim, F. Tarkanyi, R. Capote (Eds.), IAEA Technical Reports Series No. 473, IAEA, Vienna (2011)

[25] C. Kalbach, Phys Rev. C 62, 044608 (2000)

[26] O. Iwamoto, J. Nucl. Sci. Technol. 50, 409 (2013)

[27] C. Kalbach, Z. Phys. A 283, 401 (1977).

[28] S.V. Fortsch, D. Ridikas, W. Mittig, et al., J. Nucl. Sci. Technol. Supp. 2, 792 (2002)

[29] J.R. Wu, C.C. Chang, H.D. Holmgren, Phys. Rev. C 19, 370 (1979)

[30] Y. Watanabe, T. Kin, S. Araki, S. Nakayama, O. Iwamoto, presented at this conference (2016) 